\title{
Overexpression of Drosophila RFX3 Induces Apoptosis and Interferes with Differentiation of Photoreceptor Cells
}

\author{
Toshimi Sugimoto ${ }^{1}$, Takako Ueda², Hideki Yoshida ${ }^{1,3}$, Akira Murakami $^{2}$ and Masamitsu Yamaguchi ${ }^{1 *}$ \\ ${ }^{1}$ Department of Applied Biology, Kyoto Institute of Technology, Japan \\ ${ }^{2}$ Department of Bio-molecular Engineering, Kyoto Institute of Technology, Japan \\ ${ }^{3}$ Insect Biomedical Research Center, Kyoto Institute of Technology, Japan
}

\begin{abstract}
Regulatory Factor $\mathrm{X}(\mathrm{RFX})$ is a protein containing a characteristic DNA binding domain that is called RFX domain. Members of the RFX family are known to be present in human, mouse, Drosophila, Caenorhabditis elegans, Schizosaccharomyces pombe and Saccharomyces cerevisiae. Two RFX proteins, Drosophila RFX (dRFX) and dRFX2 have been identified in Drosophila so far. It is known that dRFX is involved in the differentiation of central and peripheral nerves, while dRFX2 is essential to the cell cycle progression and it may be involved in regulation of apoptosis. A novel protein, dRFX3 carrying RFX domain was identified by a Drosophila genome database search. dRFX3 is a likely homolog to the human RFX5, because of a significant sequence similarity in RFX domains between these two proteins. Two lines of transgenic fly carrying the HA-dRFX3 cDNA were established. Adult flies expressing dRFX3 specifically in the eye and the wing imaginal discs exhibited severe rough eye and atrophied wing phenotypes, respectively. 5-Bromo-2'-deoxyuridine incorporation assays and the immunological detection using anti-Cyclin B antibody indicated that the overexpression of dRFX3 in the eye imaginal discs exerted no effect on the cell cycle progressions. On the other hand, overexpression of dRFX3 in the eye imaginal discs interfered with the differentiation of R2/R5 photoreceptor cells and also induced apoptosis. These data suggest that the dRFX3 plays a negative role for the differentiation of R2/R5 photoreceptor cells and plays a positive role in the regulation of apoptosis.
\end{abstract}

\section{Introduction}

Regulatory Factor X (RFX) is a protein containing a characteristic DNA binding domain that is called RFX domain. Members of the RFX family are known to be widely present in eukaryotes [1,2], such as mammalian RFX1 to 5 in human and mouse, dRFX and dRFX2 in Drosophila, DAF-19 in C. elegance, Sak1 in S. pombe, and Crt in S. cerevisiae.

Drosophila RFX (dRFX) is homologous to human RFX1-3 (hRFX13) and to DAF-19 [3-5]. hRFX1 is a protein which binds to the X box (5'-GTNRCC $\mathrm{N}_{1-3}$ RGYAAC-3') of the Major Histocompatibility Complex (MHC) class II genes, and it is also involved in the regulation of human PCNA (proliferating cell nuclear antigen) gene promoter [6-9]. Furthermore, it is known that hRFX1 and hRFX2/hRFX3 form a heterodimer [10]. Both of them bind to the promoter region of IL$5 a$ receptor gene, and thereby regulate its expression [11]. The X-ray crystal structure shows that the RFX domain of hRFX1 is a winged helix domain. DAF-19 is a protein that regulates OSM-1, OSM-5 and OSM-6. OSM-1 and OSM-6 encode proteins of Intraflagellar Transport (IFT), and OSM-5 encodes a protein engaged in cilium assembly [1214]. dRFX is expressed in the Central Nervous System (CNS), in the Peripheral Nervous System (PNS), and in the testis during Drosophila development $[3,4]$. And the loss of dRFX is accompanied by a severe disorganization of the cilia of the sensory neuron dendrites. Thus it is known that dRFX is a transcription factor and involves in differentiation of CNS, PNS and testis $[3,4,15]$. A number of studies also indicate that RFX family proteins play major roles in regulation of genes involved in ciliogenesis $[12,16]$. However, functions of RFX family proteins other than ciliogenesis have been also reported [17].

RFX5, the most divergent mammalian member, regulates major histocompatibility class II gene expression and mutations in it results in the bare lymphocyte syndrome [17]. In addition, RFX4 has been implicated in dorsal patterning of brain development in mice and may participate in circadian rhythm regulation in humans [18-21]. dRFX2 is also reported to be involved in cell cycle progression and regulation of apoptosis. The Drosophila PCNA gene promoter contains URE (Upstream Regulatory Element), CFDD (Common Regulatory Factor for DNA Replication) and DREF (DNA Replication-Related Element Binding Factor) sites, DRE and E2F-binding sites [22-24]. A number of transcription factor such as UREF (Grainyhead: GRH), CFDD, DREF and E2F, all of which bind to their specific sites [22,25-27]. In addition to these regulatory elements, a novel regulatory element that is located between URE and DRE has been identified. dRFX2 was identified as a binding protein to this element and demonstrated that it is involved in the normal progression from G1 to $S$ phase of cell cycle and in regulation of apoptosis [28].

In addition, a novel protein, dRFX3 that contains RFX domain has been found by searching of a Drosophila genome database. In the present studies, we established transgenic fly carrying the HA-dRFX3 cDNA and utilized GAL4-UAS targeted expression system to examine effects of overexpression of HA-dRFX3 on the development [29]. Overexpression of dRFX3 in the eye imaginal discs had no effect on the cell cycle progressions of $S$ phase and $M$ phase, respectively. However it interfered with the differentiation of R2/R5 photoreceptor cells.

*Corresponding author: Masamitsu Yamaguchi, Department of Applied Biology Kyoto Institute of Technology, Matsugasaki, Sakyo-ku, Kyoto 606-8585, Japan, Tel: +81 75724 7781; E-mail: myamaguc@kit.ac.jp

Received December 31, 2012; Accepted February 20, 2013; Published March 08, 2013

Citation: Sugimoto T, Ueda T, Yoshida H, Murakami A, Yamaguchi M (2013) Overexpression of Drosophila Rfx3 Induces Apoptosis and Interferes with Differentiation of Photoreceptor Cells. J Carcinogene Mutagene 4: 137. doi:10.4172/2157-2518.1000137

Copyright: (c) 2013 Sugimoto T, et al. This is an open-access article distributed under the terms of the Creative Commons Attribution License, which permits unrestricted use, distribution, and reproduction in any medium, provided the original author and source are credited. 
Moreover overexpression of dRFX3 induced apoptosis in eye imaginal disc cells. These data suggest that the dRFX3 plays a negative role for the differentiation of R2/R5 photoreceptor cells and plays a positive role in the regulation of apoptosis.

\section{Materials and Methods}

\section{Fly stocks}

Fly stocks were maintained at $25^{\circ} \mathrm{C}$ on standard food. The yellow white fly was used as a control strain. The GAL4 driver strains and mutant strain, reaper (rpr), which were from Bloomington Stock Center or Kyoto Drosophila Genetic Resource Center. The enhancer trap lines carrying the lacZ markers X63 (inserted in rhomboid), AE127 (inserted in seven-up), and P82 (inserted in deadpan) were obtained from Y. Hiromi. These lines express the $\beta$-galactosidase markers in photoreceptor cells of R8/R2/R5, R3/R4/R1/R6, and R3/R4/R7, respectively.

\section{Establishment of transgenic flies}

$\mathrm{P}$ element-mediated germ line transformation was carried out as described earlier. F1 transformants were selected on the basis of white eye color rescue. Two independent lines carrying UAS-HA-dRFX3 on the second chromosome were used in the present studies.

\section{Scanning electron microscopy}

Adult flies were anesthetized, mounted on the stages, and observed under a Scanning Electron Microscope (SEM) VE-7800 (Keyence Inc.) in the low vacuum mode. In every experiment, the eye phenotype of at least five adult flies of each line was simultaneously examined by scanning electron microscopy, and these experiments were repeated 3 times. In the experiments, no significant variation in eye phenotype among the five individuals was observed.

\section{5-Bromo-2'-deoxyuridine (BrdU) labeling}

Detection of cells in S phase was performed using a BrdU-labeling method as described previously with minor modifications [30-33]. Third instar larvae were dissected in Drosophila Ringer's solution and the eye imaginal discs were suspended in Grace's insect medium, then incubated in the presence of $75 \mu \mathrm{g} / \mathrm{ml} \mathrm{BrdU}$ (Roche) at $25^{\circ} \mathrm{C}$ for $30 \mathrm{~min}$. The samples were fixed in Carnoy's fixative (ethanol/acetic acid/chloroform, $6: 1: 3$ ) at $25^{\circ} \mathrm{C}$ for $20 \mathrm{~min}$, and further fixed in $80 \%$ ethanol/50 $\mathrm{mM}$ glycine buffer $(\mathrm{pH} 2.0)$ at $-20^{\circ} \mathrm{C}$ for $16 \mathrm{~h}$. Incorporated $\mathrm{BrdU}$ was visualized using an anti-BrdU antibody and an anti-mouse Alexa 594 antibody (Invitrogen) at $25^{\circ} \mathrm{C}$ for $2 \mathrm{~h}$.

\section{Immunohistology}

Third instar larvae were dissected in Drosophila Ringer's solution and the eye imaginal discs were fixed in $4 \%$ paraformaldehyde/PBS (Phosphate-Buffered Saline) at $25^{\circ} \mathrm{C}$ for $30 \mathrm{~min}$. After washing with PBS, the samples were blocked with $10 \%$ normal goat serum in PBST (PBS containing $0.15 \%$ Triton $\mathrm{X}-100$ ) at $25^{\circ} \mathrm{C}$ for $20 \mathrm{~min}$. Then the samples were incubated with culture supernatant of hybridoma cells producing mouse anti-HA monoclonal antibody (supplied by Dr. M. Inagaki) at a 1:100 dilution, mouse anti- $\beta$-galactosidase monoclonal antibody (Promega) at a 1:500 dilution or rabbit anti-Cyclin B polyclonal antibody (Santa Cruz Biotechnology, Inc.) at a 1:200 dilution at $4^{\circ} \mathrm{C}$ for $16 \mathrm{~h}$. After washing with PBST, the samples were incubated with the second antibody, an alkaline phosphatase-conjugated goat anti-mouse Immunoglobulin G (IgG) (Promega) at a 1:500 dilution or anti-rabbit Alexa 594 antibody (Invitrogen) at a 1:400 dilution at $25^{\circ} \mathrm{C}$ for $2 \mathrm{~h}$.
After washing with PBST, the samples were mounted and analyzed or the color was developed in a solution containing NBT $(100 \mathrm{mM}$ Tris- $\mathrm{HCl} \mathrm{pH}$ 9.5, $100 \mathrm{mM} \mathrm{NaCl}, 5 \mathrm{mM} \mathrm{MgCl}, 0.34 \mathrm{mg} / \mathrm{ml}$ nitroblue tetrazolium salt), and BCIP $(0.175 \mathrm{mg} / \mathrm{ml}$ 5-bromo-4-chloro-3-indolyl phosphate toluidinium salt). The samples were washed with PBS and mounted in $90 \%$ glycerol/PBS for microscopic observation. In the case of immuno-staining photoreceptor cells, the F1 third instar larvae from the cross of GMR-GAL4; +, or GMR-GAL4; UAS-HA-dRFX3 with each enhancer trap line were dissected.

\section{Detection of apoptotic cells}

The third instar larvae were dissected and the eye imaginal discs were immunostained. The primary antibody, rabbit anti-cleaved caspase-3 polyclonal antibody (Cell Signaling Technology) was used at a 1:200 dilution at $4^{\circ} \mathrm{C}$ for $16 \mathrm{~h}$. The second antibody, anti-rabbit Alexa 594 antibody (Invitrogen) was used at $25^{\circ} \mathrm{C}$ for $2 \mathrm{~h}$. After washing with PBST, the samples were mounted and analyzed.

\section{Quantification of the data}

Intensities of immunofluorescent signals were quantified with Image J software. Relative area size of the adult compound eyes were also quantified with Image J software.

\section{Results and Discussion}

\section{Overexpression of dRFX3 disrupts normal development in Drosophila}

Based on a comparison of the RFX domain between human and Drosophila, dRFX3 shows the highest homology with hRFX5 (46.5\% identity and 67\% similarity) (Figure 1). hRFX1 and hRFX2/hRFX3 form a heterodimer, both of which bind to the promoter region of IL$5 a$ receptor gene, and thereby regulate its expression $[9,10]$. However, hRFX5 has no dimerization motifs as well as dRFX3. Therefore dRFX3 may have similar functions to hRFX5. To investigate the effects of overexpression of dRFX3 in living flies, we utilized the GAL4-UAS targeted expression system. Two independent transgenic lines, strain 18 and strain 53 carrying UAS-dRFX3 with HA-tag (UAS-HA-dRFX3) were established, and crossed them with 40 lines of GAL4 drivers expressing GAL4 in specific tissues and developmental stages in Drosophila.

The sd-GAL4>HA-dRFX3, MS1096-GAL4>HA-dRFX3, enGAL4>HA-dRFX3, vg-GAL4>HA-dRFX3, 48Y-GAL4>HA-dRFX3, arm-GAL4>HA-dRFX3 and 645-GAL4>HA-dRFX3 induced abnormal wing phenotypes such as atrophied, crumpled or unclosed wing phenotypes, respectively (Figure 2A) (Table 1). When the pnrGAL4 that expressed GAL4 at presumptive notum region in wing discs was crossed with UAS-HA-dRFX3, split thorax phenotype was induced (Figure 2B) (Table 1). Overexpression of dRFX3 with GMRGAL4 (GMR-GAL4>HA-dRFX3) and eyeless-GAL4 (eyeless-GAL4> $H A-d R F X 3)$ drivers induced rough eye and small eye phenotypes, respectively (Figure 3) (Table 1). Size of the adult compound eye (eyelessGAL4> HA-dRFX3) was reduced to $93 \%$ with strain 18 and $70 \%$ with strain 53 in compared to the control eyeless-GAL4 flies. It is known that 389-GAL4 driver strain express GAL4 in embryonic CNS. When 389GAL4 was crossed with $U A S-H A-d R F X 3$, multiple phenotypes such as rough eye, small head, unclosed wing and crumpled wing phenotypes were induced (Table 1). Altogether the overexpression using strain 53 of transgenic fly carrying the HA-dRFX3 cDNA exhibited severer phenotypes than the strain 18 . This is very likely due to the higher expression of HA-dRFX3 in strain 53 than in strain 18 (Figure 3, 
Citation: Sugimoto T, Ueda T, Yoshida H, Murakami A, Yamaguchi M (2013) Overexpression of Drosophila Rfx3 Induces Apoptosis and Interferes with Differentiation of Photoreceptor Cells. J Carcinogene Mutagene 4: 137. doi:10.4172/2157-2518.1000137

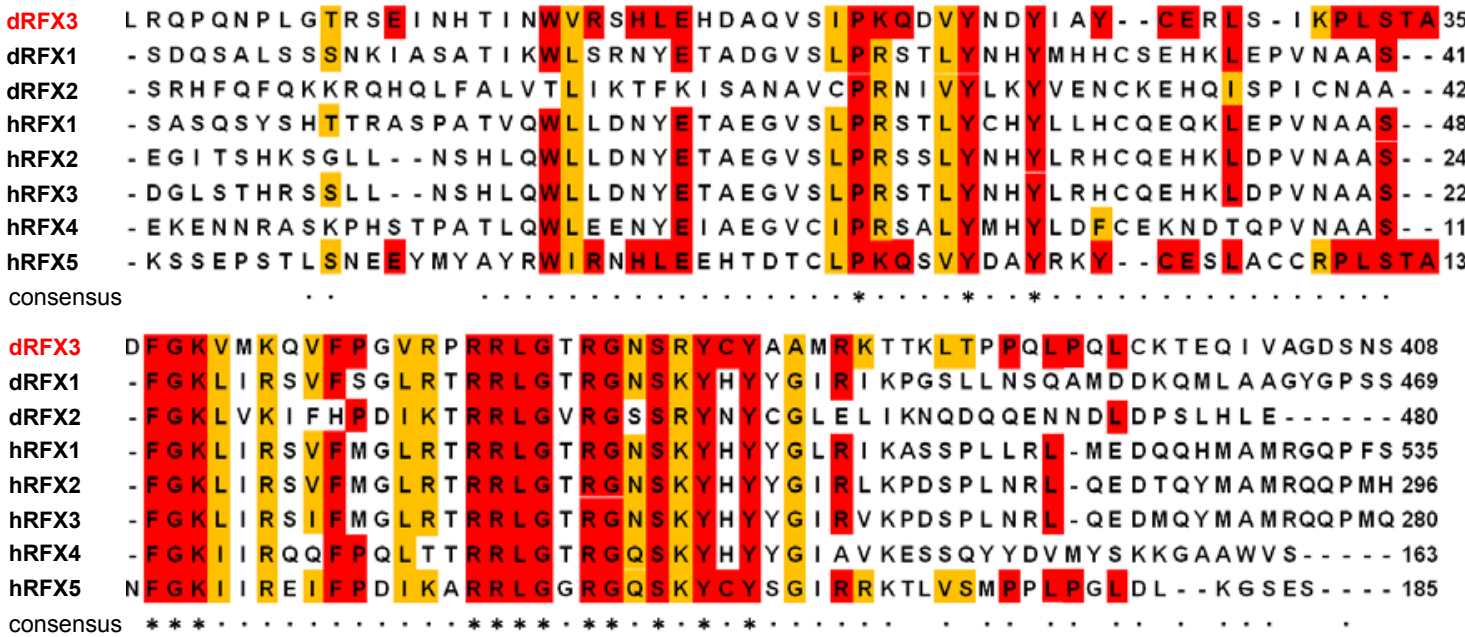

Figure 1: Multiple sequence alignment of RFX domains. Multiple sequence alignment of the conserved RFX domain between human and Drosophila RFX family proteins. Red and yellow boxes represent identical and similar amino acids between dREF3 and hRFX5.

A

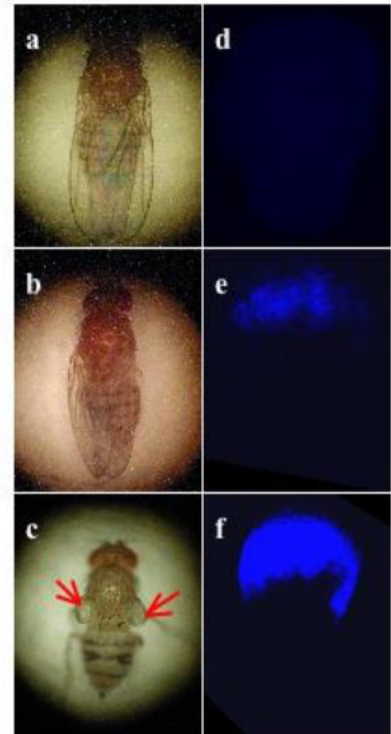

B

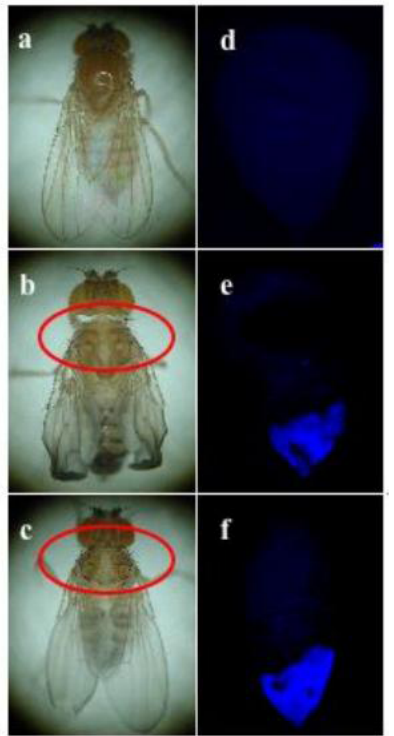

Figure 2: Adult flies expressing dRFX3 in wing imaginal disc exhibited atrophied wing and split thorax phenotypes. (a) $\sim(\mathrm{c})$ Micrographs of adult flies. (d) (f) Immunostainning of wing imaginal discs with anti-HA antibody. (A) (a, d) sd-GAL4/+; +/+, (b, e) sd-GAL4/+; UAS-HA-dRFX3 (strain 18)/+, (c, f) sd-GAL4/+; UAS-HA-d RFX3 (strain 53)/+, (B) (a, d) +/+; pnr-GAL4/+, (b, e) +/+; pnr-GAL4/UAS-HA-dRFX3 (strain 18)/+, (c, f) +/+; pnr-GAL4/UAS-HA-dRFX3 (strain 53) /+. These flies were developed at $28^{\circ} \mathrm{C}$. HA signals are detected in ventral regions (A-e and A-f) or dorsal regions (B-e and B-f) of wing discs. Arrows indicate the atrophied wing Red-circled regions indicate the split thorax of the adult fly.

Panels A-g and B-g). The other 15 GAL4 drivers exerted no detectable abnormality (Table 1). Since cell cycle regulation and differentiation have been well characterized during eye development in Drosophila, we focused on analyses of the rough eye phenotype induced by dRFX3 in the following studies.

Overexpression of dRFX3 in eye imaginal discs exerts no effect on the cell cycle progression.

In third instar larvae, the Morphogenetic Furrow (MF) appears at the end of posterior in the eye imaginal discs, and then it moves to the anterior direction. Cells in the anterior to the MF proliferate asynchronously. Once those cells are on MF, they are arrested synchronously at G0/G1 phase. Cells in the posterior to MF undergo one more cell cycle such as S, G2, M, which in turn differentiate into photoreceptors of the adult ommatidium [30].

In GMR-GAL4, it is known that GAL4 is expressed in the region within and posterior to MF by GMR carrying transcription factor Glass-binding sites. The flies with GMR-GAL4>HA-dRFX3 showed specific expression of HA-dRFX3 in the region posterior to MF that was detected by immunostaining with anti-HA antibody (Figures $3 \mathrm{~A}$ and $3 \mathrm{~B}(\mathrm{~d}-\mathrm{f})$ ).

The rough eye phenotype may arise from the ectopic induction or arrest of cell cycle in the eye imaginal discs. To examine effect of dRFX3 overexpression on DNA synthesis in the eye imaginal disc cells, at first, we carried out the BrdU incorporation assay that can detect 


\begin{tabular}{|c|c|c|c|c|}
\hline GAL4 line & Chromosome linkage & Expression pattern & Lethal phase & Phenotype \\
\hline GMR(\#16) & $\mathrm{x}$ & eye & & rough eye \\
\hline GMR & II & eye & & rough eye \\
\hline eyeless & II & eye & & rough eye \\
\hline eyeless/CyO & II & eye & & rough eye \\
\hline sev/CyO & II & eye & larval,pupal & \\
\hline$s d$ & $x$ & wing & & atrophied wing(\#53) \\
\hline MS1096 & $\mathrm{x}$ & wing & & crumpled wing(\#18), atrophied wing(\#53) \\
\hline $\mathrm{Hs} / \mathrm{CyO}$ & II & wing, nerve & & no effect \\
\hline Hs & II & wing, nerve & & no effect \\
\hline en & II & wing, imaginal discs & embryo(\#53) & crumpled wing(\#18) \\
\hline en/CyO & II & wing, imaginal discs & embryo(\#53) & unclosed wing(\#18) \\
\hline ve & II & wing & & no effect \\
\hline$v g$ & II & wing & & crumpled or atrophied wing \\
\hline $\mathrm{ap} / \mathrm{CyO}$ & II & wing, nerve & embryo & \\
\hline$w g$ & II & wing & & no effect \\
\hline pnr/TM3 & II & wing & & split back \\
\hline elav & $x$ & embryonic nerve system & & no effect \\
\hline 389 & II & embryonic CNS & & rough eye,small head,unclosed wing,crumpled wing $(\# 53)$ \\
\hline $91 Y$ & II & CNS & & no effect \\
\hline twist & $x$ & mesoderm & & low viability(\#53) \\
\hline $48 Y$ & II & endoderm & & unclosed wing(\#53) \\
\hline$d p p / C y O$ & II & endo/mesoderm & & no effect \\
\hline dII & II & early embryo & & no effect \\
\hline dII/CyO & II & early embryo & & no effect \\
\hline arm & II & segments, ubiquitous & & unclosed wing(\#53) \\
\hline $\mathrm{Cg}$ & II & blood cells & larval & \\
\hline $\mathrm{Hml}$ & II & larval hemocyte & & no effect \\
\hline c323a & $x$ & SG, gut, embryo, follicle cells & larval & \\
\hline$T 155$ & II & follicle cells & embryo(\#53) & low viability(\#18) \\
\hline $55 B$ & II & follicle cells & & no effect \\
\hline $198 Y$ & II & follicle cells, oocyte & & no effect \\
\hline 645 & II & oocyte & & crumpled wing(\#53) \\
\hline $69 B$ & $x$ & larval discs & & no effect \\
\hline T80/CyO & II & imaginal discs & larval & \\
\hline Act25C/CyO-GFP & II & imaginal discs & larval & \\
\hline Act5C/TM6B & II & all tissues & & crooked body(\#53) \\
\hline$d a$ & II & ubiquitous & & no effect \\
\hline kr/TM3 & II & embryo & & no effect \\
\hline$h h / T M 6 B$ & II & embryo & & low viability \\
\hline tub/TM3 & II & all tissues & larval & \\
\hline
\end{tabular}

Table 1: Summary of effects of HA-dRFX3 expression with each GAL4 driver lines.

the cells in $\mathrm{S}$ phase (Figure $4 \mathrm{~A}$ ). In eye discs expressing GAL4 alone, BrdU incorporation was observed in the whole anterior region to the $\mathrm{MF}$ and in a line just posterior to the MF, which cannot discriminate from the pattern of BrdU incorporation in wild-type fly discs (Figure $4 \mathrm{~A}(\mathrm{a}))$. In the eye imaginal discs overexpressing dRFX3, the pattern of $\mathrm{BrdU}$ incorporation was indistinguishable from the one expressing GAL4 alone (Figure 4A). The quantification of BrdU-positive cells also indicates no significant difference between control and dRFX3overexpressing flies.

Start of M phase is regulated by the phosphorylation of MPF (M-Phase Promoting Factor) that is comprised of Cyclin B and Cdc2. And the Cyclin B is abolished at the end of M phase. We therefore carried out the immunological detection of Cyclin B with anti-Cyclin $\mathrm{B}$ antibody to examine the effect of dRFX3 overexpression on the progression of $\mathrm{M}$ phase (Figure $4 \mathrm{~B}$ ). In eye discs expressing GAL4 alone, Cyclin B signal was detected in the whole anterior region to the MF and in a line posterior to the MF, which cannot discriminate from the pattern of wild-type fly discs (Figure 4B (a)). In the eye imaginal discs overexpressing dRFX3, the pattern of Cyclin B signal was indistinguishable from the one expressing GAL4 alone (Figure $4 \mathrm{~B})$. These results suggest that the overexpression of dRFX 3 in the eye imaginal discs exerts no effect on the cell cycle progression of $S$ phase and $\mathrm{M}$ phase.

\section{Overexpression of $\mathrm{dRFX} 3$ in eye imaginal discs interferes with the differentiation of $\mathrm{R} 2 / \mathrm{R} 5$ photoreceptor cells}

In Drosophila development, photoreceptor cells are generated in specific order with the MF crosses the eye imaginal discs from posterior to anterior: R8 photoreceptor is generated first after passing of the MF, then R2/R5, R3/R4, R1/R6, and R7 that is the last cell, all of which are generated sequentially [31,32]. Thereafter, each photoreceptor cell forms rhabdomere that receives light and then transduces it to chemical signal.

The rough eye phenotype may arise from the interference of 
A
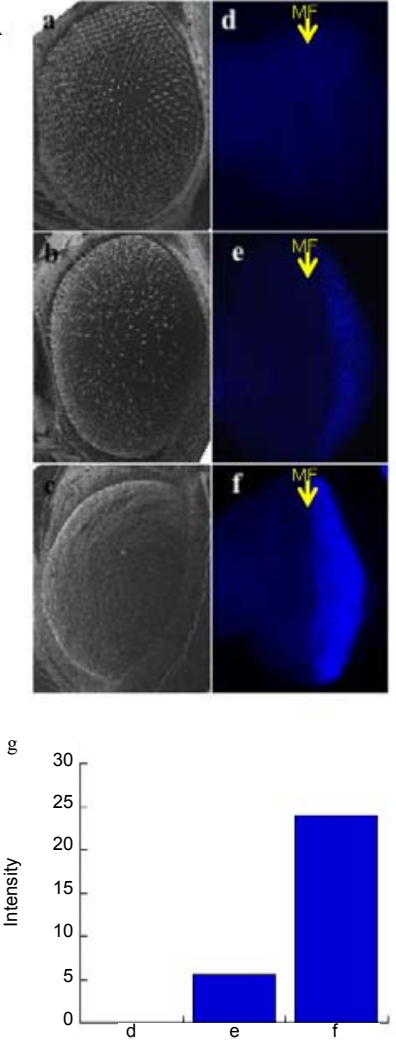

B

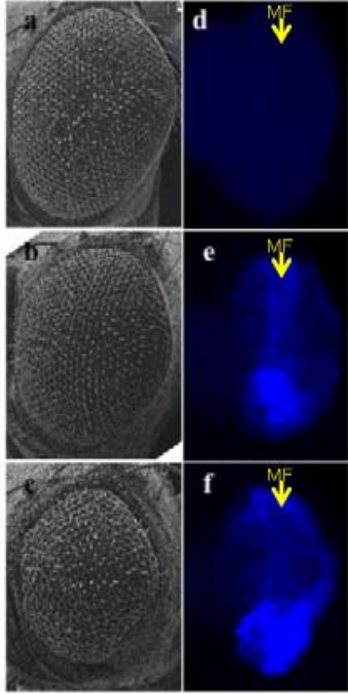

g

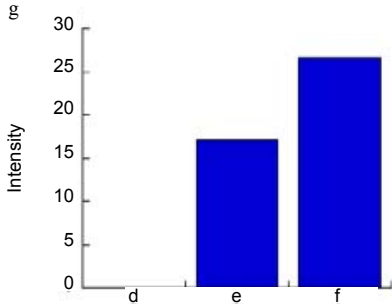

Figure 3: Adult flies expressing dRFX3 in eye imaginal disc exhibited rough eye phenotype. (a) (c) Scanning electron micrographs of adult compound eyes. (d) (f) Immuno-stainning of eye imaginal discs with anti-HA antibody. (A) (a, d) GMR-GAL4/+; +/+, (b, e) GMR-GAL4/+; UAS-HA-dRFX3 (strain 18) /+, (c, f) GMR-GAL4/+; UAS-HA-dRFX3 (strain 53) /+, (B) (a, d) +/+; eyelessGAL4/+, (b, e) +/+; eyeless-GAL4/UAS-HA-dRFX3 (strain 18) /+, (c, f) +/+; eyeless-GAL4/UAS-HA-dRFX3 (strain 53) /+. These flies were developed at $28^{\circ} \mathrm{C}$. Arrows represent the morphogenetic furrow (MF). Intensities of HA signals are quantified and shown in lower panels $(\mathrm{g})$, confirming higher expression of HA-dRFX3 in strain 53 than in strain 18. Averaged values from several discs are shown.

photoreceptor cell differentiation in the eye imaginal discs. We therefore carried out the immunological detection of photoreceptor cells using enhancer trap lines expressing a nucleus-localized form of $\beta$-galactosidase markers in photoreceptor cells. In this analysis, we utilized three enhancer trap lines, X63, AE127 and p82, specifically expressing $\beta$-galactosidase in $\mathrm{R} 8 / \mathrm{R} 2 / \mathrm{R} 5, \mathrm{R} 3 / \mathrm{R} 4 / \mathrm{R} 1 / \mathrm{R} 6$ and $\mathrm{R} 3 / \mathrm{R} 4 /$ R7, respectively. The eye imaginal discs of F1 third instar larvae from the cross of GMR-GAL4; +, or GMR-GAL4; UAS-HA-dRFX3 with each enhancer trap line were dissected, and then immunostained with anti- $\beta$-galactosidase antibody (Figures $5 \mathrm{~A}-\mathrm{I}$ ). In eye imaginal discs overexpressed dRFX3, only R8 photoreceptor cell was stained by cross with X63 enhancer trap line (Figures 5B and 5C). On the other hand, when the enhancer trap lines AE127 and p82 were used, none of the photoreceptor cells was stained (Figures 5E, F, H and I). These results indicate that the overexpression of dRFX 3 in the eye imaginal discs interfere with the differentiation of R2/R5 and consequently the following R3/R4, R1/R6 and R7 differentiation, but exerted no effect on that of R8. Therefore overexpression of dRFX3 may have disturbed expression of genes responsible for differentiation of R2/R5 photoreceptor cells, although further analyses are necessary to identify the responsible genes. Genetic screen to identify mutations that can suppress the rough eye phenotype induced by dRFX3-overexpression

may allow us to identify such genes. In any event, the transgenic flies established in this study would be useful to perform such genetic screen.

Overexpression of dRFX3 in eye imaginal discs induces caspase-dependent apoptosis

Overexpression of dRFX3 in the eye imaginal discs interfered with the differentiation of photoreceptor cells. Since there is a possibility that interference of differentiation process lead to apoptosis, we investigated whether apoptosis is induced in the eye imaginal discs, by immunological detection of the activated caspase-3. In this assay, GMRGAL4/+; GMR-rpr that is expressing GAL4 and Reaper, a Drosophila cell death factor, was used for a positive control. And GMR-GAL4/+ flies expressing GAL4 alone was used for a negative control. In the eye imaginal discs of GMR-GAL4/+, cell death was barely detectable (Figure 6b). In contrast, innumerable apoptotic signals were detected in the region within and posterior to the MF in the eye imaginal discs of GMR-GAL4/+; GMR-rpr (Figure 6a). When dRFX3 was overexpressed in the eye imaginal discs, a large number of apoptotic signals were detected in the region posterior to the MF (Figures $6 \mathrm{c}$ and $6 \mathrm{~d}$ ). Furthermore, when the flies overexpressing dRFX 3 were crossed with the flies expressing the Caspase inhibitors such as P35 and DIAP1, the rough eye phenotypes were effectively suppressed in their progenies (Figures 7A and 7B (a,b,d,e,g,h)) and the apoptosis signals detected in $\mathrm{dRFX} 3$-overexpression (Figures $7 \mathrm{~A}(\mathrm{c})$ and $7 \mathrm{~B}(\mathrm{c})$ ) also disappeared from their eye imaginal discs (Figures $7 \mathrm{~A}(\mathrm{f}, \mathrm{i})$ and $7 \mathrm{~B}(\mathrm{f}, \mathrm{i})$ ). These results indicate that overexpression of $\mathrm{dRFX} 3$ in eye imaginal discs induces the Caspase-dependent apoptosis.

Taken together, our data suggests that the dRFX3 plays a negative role for the differentiation of R2/R5 photoreceptor cells and plays a positive role in the regulation of apoptosis. A number of studies indicate that RFX family proteins play major roles in regulation of genes involved in ciliogenesis $[12,16]$. However, this study shows
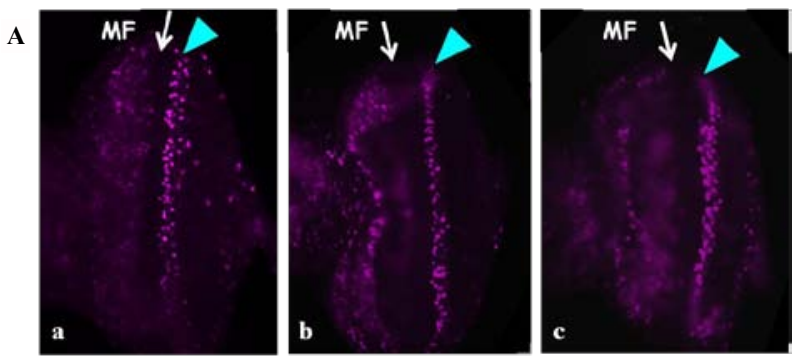

B
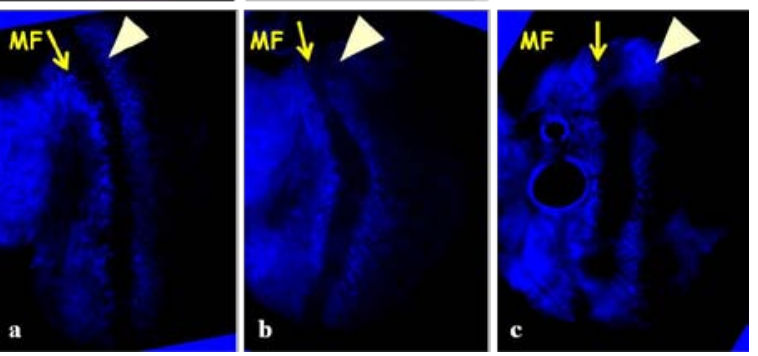

Figure 4: Overexpression of HA-dRFX3 in eye imaginal disc exerted no effect on the cell cycle progression. (A) After incorporation of BrdU, the eye imaginal discs were immuno-stained with anti-BrdU antibody. (a) GMR-GAL4/+; +/+, (b) GMR-GAL4/+; UAS-HA-dRFX3 (strain 18) /+, (c) GMR-GAL4/+; UASHA-dRFX3 (strain 53)/+. (B) Immunostaining of eye discs with anti-Cyclin B antibody. (a) GMR-GAL4/+; +/+, (b) GMR-GAL4/+; UAS-HA-dRFX3 (strain 18)/+, (c) GMR-GAL4/+; UAS-HA-dRFX3 (strain 53) /+. Arrows indicate morphogenetic furrow (MF). Blue arrowheads indicate BrdU-positive cells behind MF and white arrowheads indicate Cyclin B-positive cells behind MF. 


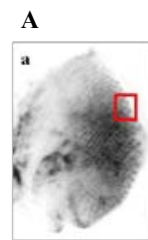

D

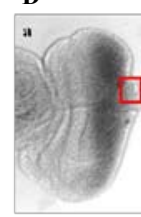

G
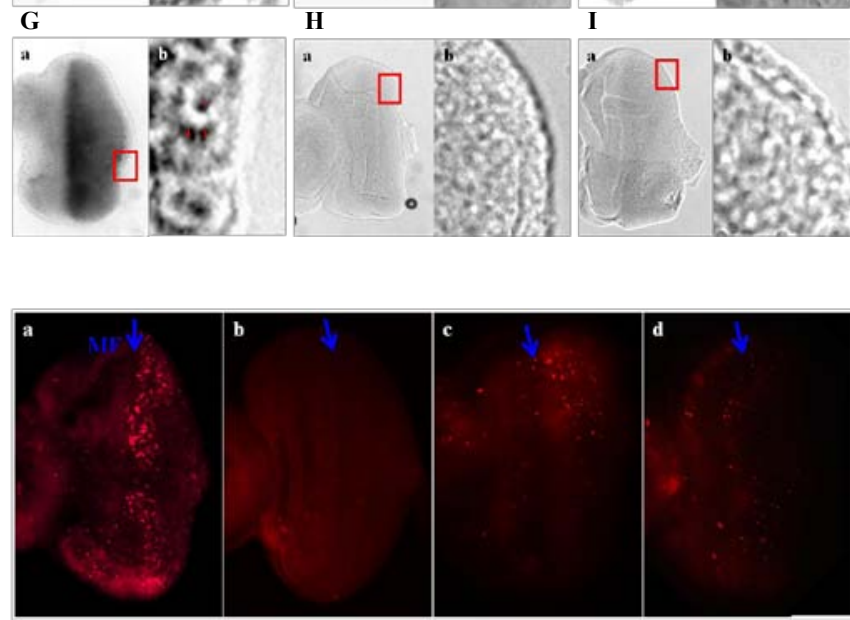

Figure 5: Overexpression of dRFX3 in the eye imaginal discs interfered with the differentiation of R2/R5, R1/R6 and R7 differentiation, but exerted no effect on that of R8. Males of the strain X63 were crossed with females of GMR-GAL4; + or GMR-GAL4; UAS-HA-dRFX3. (A) GMR-GAL4/+; +; X63/+, (B) GMR-GAL4/+; UAS-HA-dRFX3 (strain 18) /+; X63/+, (C) GMRGAL4/+; UAS-HA-dRFX3 (strain 53) /+; X63/+. The X63 expresses lacZ in R8 and R2/R5. Males of the strain AE127 were crossed with females of GMRGAL4; + or GMR-GAL4; UAS-HA-dRFX3. (D) GMR-GAL4/+; +; AE127/+, (E) GMR-GAL4/+; UAS-HA-dRFX3 (strain 18) /+; AE127/+, (F) GMR-GAL4/+; UAS-HA-dRFX3 (strain 53) /+; AE127/+. The AE127 expresses lacZ in R3/ R4 and R1/R6. Males of the strain p82 were crossed with females of GMRGAL4; + or GMR-GAL4; UAS-HA-dRFX3. (G) GMR-GAL4/+; +; p82/+, (H) GMR-GAL4/+; UAS-HA-dRFX3 (strain 18) /+; p82/+, (I) GMR-GAL4/+; UASHA-dRFX3 (strain 53) /+; p82/+. The p82 expresses lacZ in R3/R4 and R7. The eye imaginal discs of these progenies were immunostained with anti$\beta$-galactosidase antibody.
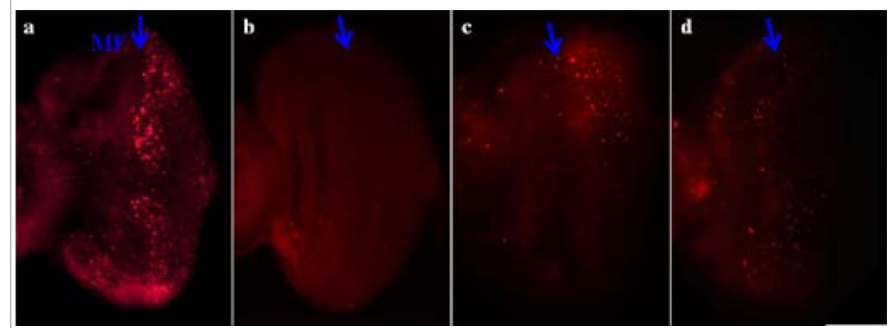

Figure 6: Ectopic expression of dRFX3 in eye imaginal discs induced apoptosis. Detection of apoptotic cells in eye imaginal discs by immunostaining with anti-cleaved Caspase 3 antibody. (a) GMR-GAL4/+; GMR-rpr, (b) GMRGAL4/+; +/+, (c) GMR-GAL4/+; UAS-HA-dRFX3 (strain 18) /+, (d) GMRGAL4/+; UAS-HA-dRFX3 (strain 53). Arrows represent the Morphogenetic Furrow (MF).
A
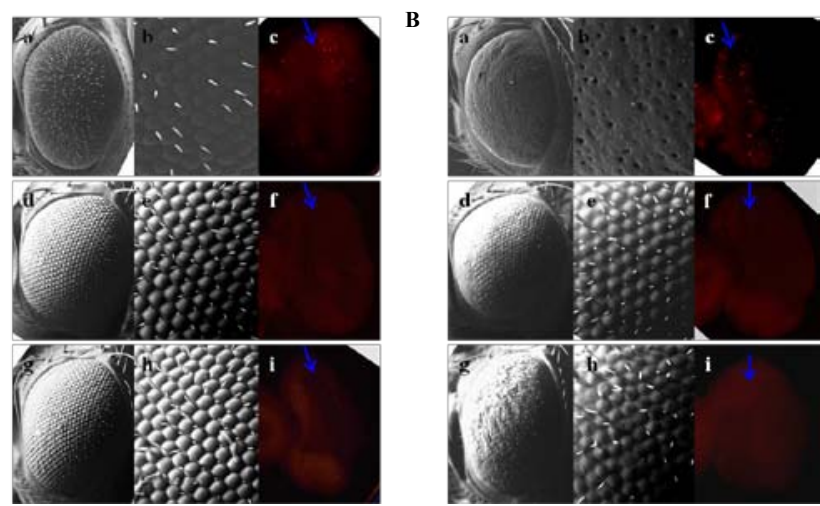

Figure 7: Coexpressions of dRFX3 and Caspase inhibitors such as P35 and DIAP1, which could be rescued the rough eye phenotypes induced by HAdRFX3. (A) In the case of overexpression of HA-dRFX3 (strain 18), (B) In the case of overexpression of HA-dRFX3 (strain 53). (a c) GMR-GAL4/+; UASHA-dRFX3, (d f) GMR-GAL4/+; UAS-HA-dRFX3/+; UAS-p35/+, (g i) GMRGAL4/+; UAS-HA-dRFX3/GMR-DIAP1. (a, b, d, e, g, h) Scanning electron micrographs of adult compound eyes. (c, $f, i)$ Detection of apoptotic cells in eye imaginal discs by immunostaining with anti-cleaved Caspase 3 antibody. Arrows represent the Morphogenetic Furrow (MF).

that the dRFX3, a homolog of RFX5 has other important function in Drosophila. Involvement of dRFX3 in apoptosis also implicates possible links with mutagenesis and cancer in which apoptosis plays critical roles.

\section{References}

1. Emery P, Durand B, Mach B, Reith W (1996) RFX proteins, a novel family of DNA binding proteins conserved in the eukaryotic kingdom. Nucleic Acids Res 24: 803-807.

2. Laurençon A, Dubruille R, Efimenko E, Grenier G, Bissett R, et al. (2007) Identification of novel regulatory factor $X(R F X)$ target genes by comparative genomics in Drosophila species. Genome Biol 8: R195.

3. Durand B, Vandaele C, Spencer D, Pantalacci S, Couble P (2000) Cloning and characterization of dRFX, the Drosophila member of the RFX family of transcription factors. Gene 246: 285-293.

4. Dubruille R, Laurençon A, Vandaele C, Shishido E, Coulon-Bublex M, et al. (2002) Drosophila regulatory factor $X$ is necessary for ciliated sensory neuron differentiation. Development 129: 5487-5498.

5. Lubelsky Y, Reuven N, Shaul Y (2005) Autorepression of rfx1 gene expression: functional conservation from yeast to humans in response to DNA replication arrest. Mol Cell Biol 25: 10665-10673.

6. Liu M, Lee BH, Mathews MB (1999) Involvement of RFX1 protein in the regulation of the human proliferating cell nuclear antigen promoter. J Biol Chem 274: 15433-15439

7. Labrie C, Lee BH, Mathews MB (1995) Transcription factors RFX1/EF-C and ATF-1 associate with the adenovirus E1A-responsive element of the human proliferating cell nuclear antigen promoter. Nucleic Acids Res 23: 3732-3741.

8. Reith W, Herrero-Sanchez C, Kobr M, Silacci P, Berte C, et al. (1990) MHC class II regulatory factor RFX has a novel DNA-binding domain and a functionally independent dimerization domain. Genes Dev 4: 1528-1540.

9. Reinhold W, Emens L, Itkes A, Blake M, Ichinose I, et al. (1995) The myc intron-binding polypeptide associates with RFX1 in vivo and binds to the majo histocompatibility complex class II promoter region, to the hepatitis B virus enhancer, and to regulatory regions of several distinct viral genes. Mol Cell Biol 15: 3041-3048.

10. Reith W, Ucla C, Barras E, Gaud A, Durand B, et al. (1994) RFX1, a transactivato of hepatitis $B$ virus enhancer I, belongs to a novel family of homodimeric and heterodimeric DNA-binding proteins. Mol Cell Biol 14: 1230-1244.

11. Iwama A, Pan J, Zhang P, Reith W, Mach B, et al. (1999) Dimeric RFX proteins contribute to the activity and lineage specificity of the interleukin- 5 receptor 
Citation: Sugimoto T, Ueda T, Yoshida H, Murakami A, Yamaguchi M (2013) Overexpression of Drosophila Rfx3 Induces Apoptosis and Interferes with Differentiation of Photoreceptor Cells. J Carcinogene Mutagene 4: 137. doi:10.4172/2157-2518.1000137

alpha promoter through activation and repression domains. Mol Cell Biol 19: 3940-3950.

12. Swoboda P, Adler HT, Thomas JH (2000) The RFX-type transcription factor DAF-19 regulates sensory neuron cilium formation in C. elegans. Mol Cell 5 : 411-421.

13. Cole DG, Diener DR, Himelblau AL, Beech PL, Fuster JC, et al. (1998) Chlamydomonas kinesin-II-dependent intraflagellar transport (IFT): IFT particles contain proteins required for ciliary assembly in Caenorhabditis elegans sensory neurons. J Cell Biol 141: 993-1008.

14. Collet J, Spike CA, Lundquist EA, Shaw JE, Herman RK (1998) Analysis of osm-6, a gene that affects sensory cilium structure and sensory neuron function in Caenorhabditis elegans. Genetics 148: 187-200.

15. Vandaele C, Coulon-Bublex M, Couble P, Durand B (2001) Drosophila regulatory factor $\mathrm{X}$ is an embryonic type I sensory neuron marker also expressed in spermatids and in the brain of Drosophila. Mech Dev 103: 159-162.

16. Li JB, Gerdes JM, Haycraft CJ, Fan Y, Teslovich TM, et al. (2004) Comparative genomics identifies a flagellar and basal body proteome that includes the BBS5 human disease gene. Cell 117: 541-552.

17. Reith W, Mach B (2001) The bare lymphocyte syndrome and the regulation of MHC expression. Annu Rev Immunol 19: 331-373.

18. Blackshear PJ, Graves JP, Stumpo DJ, Cobos I, Rubenstein JL, et al (2003) Graded phenotypic response to partial and complete deficiency of a brain-specific transcript variant of the winged helix transcription factor RFX4. Development 130: 4539-4552.

19. Zarbalis K, May SR, Shen Y, Ekker M, Rubenstein JL, et al. (2004) A focused and efficient genetic screening strategy in the mouse: identification of mutations that disrupt cortical development. PLoS Biol 2: E219.

20. Araki R, Takahashi H, Fukumura R, Sun F, Umeda N, et al. (2004) Restricted expression and photic induction of a novel mouse regulatory factor X4 transcrip in the suprachiasmatic nucleus. J Biol Chem 279: 10237-10242.

21. Zhang D, Zeldin DC, Blackshear PJ (2007) Regulatory factor X4 variant 3: a transcription factor involved in brain development and disease. J Neurosci Res 85: 3515-3522.

22. Yamaguchi M, Hayashi Y, Matsukage A (1995) Essential role of E2F recognition sites in regulation of the proliferating cell nuclear antigen gene promoter during Drosophila development. J Biol Chem 270: 25159-25165.
23. Hirose F, Yamaguchi M, Handa H, Inomata Y, Matsukage A (1993) Nove 8-base pair sequence (Drosophila DNA replication-related element) and specific binding factor involved in the expression of Drosophila genes for DNA polymerase alpha and proliferating cell nuclear antigen. J Biol Chem 268 2092-2099.

24. Yamaguchi M, Hayashi Y, Nishimoto Y, Hirose F, Matsukage A (1995) A nucleotide sequence essential for the function of DRE, a common promoter element for Drosophila DNa replication-related genes. J Biol Chem 270: 15808 15814.

25. Hayashi Y, Hirose F, Nishimoto Y, Shiraki M, Yamagishi M, et al. (1997) Identification of CFDD (common regulatory factor for DNA replication and DREF genes) and role of its binding site in regulation of the proliferating cell nuclear antigen gene promoter. J Biol Chem 272: 22848-22858.

26. Cuvier O, Hart CM, Käs E, Laemmli UK (2002) Identification of a multicopy chromatin boundary element at the borders of silenced chromosomal domains. Chromosoma 110: 519-531.

27. Hayashi Y, Yamagishi M, Nishimoto Y, Taguchi O, Matsukage A, et al. (1999) A binding site for the transcription factor Grainyhead/Nuclear transcription factor-1 contributes to regulation of the Drosophila proliferating cell nuclear antigen gene promoter. J Biol Chem 274: 35080-35088.

28. Otsuki K, Hayashi Y, Kato M, Yoshida H, Yamaguchi M (2004) Characterization of dRFX2, a novel RFX family protein in Drosophila. Nucleic Acids Res 32 5636-5648.

29. Brand AH, Perrimon N (1993) Targeted gene expression as a means of altering cell fates and generating dominant phenotypes. Development 118: 401-415.

30. Wolff T, Ready FD (1993) In the development of Drosophila melanogaster Cold Spring Harbor Laboratory Press: 1277-1326.

31. Heberlein U, Moses K (1995) Mechanisms of Drosophila retinal morphogenesis: the virtues of being progressive. Cell 81: 987-990.

32. Freeman M (1997) Cell determination strategies in the Drosophila eye Development 124: 261-270.

33. Suyari O, Kawai M, Ida H, Yoshida H, Sakaguchi K, et al. (2012) Differentia requirement for the $\mathrm{N}$-terminal catalytic domain of the DNA polymerase Î $\mathrm{\mu}$ p255 subunit in the mitotic cell cycle and the endocycle. Gene 495: 104-114. 